\title{
Spectrum of interstitial lung disease in China from 2000 to 2012
}

\author{
To the Editor:
}

Epidemiological data on interstitial lung disease (ILD) are very limited in China. This study describes the distribution of ILD in China based on data from a large ILD centre.

All patients admitted to the Dept of Pulmonary and Critical Care Medicine of Beijing Chao-Yang Hospital (affiliated to Capital Medical University, Beijing, China) for further diagnosis of suspected ILD from January 1, 2000 to December 31, 2012 were included in the analysis.

Patients suspected with ILD underwent a standard investigation protocol as described previously [1]. The diagnosis of ILD and idiopathic interstitial pneumonia (IIP) was based on the international ILD guidelines and the American Thoracic Society/European Respiratory Society international consensus classification of IIP [2, 3]. Clinical data were recorded, including demographic information, environmental and occupational exposure, symptoms, physical examination, laboratory tests, pulmonary function tests, arterial blood gas analysis, imaging and pathology if available.

The study protocol was approved by the Institutional Human Ethics Review Committee. Statistical analyses were performed with SPSS 18.0 (SPSS Inc., Chicago, IL, USA). Normally distributed continuous variables are presented as mean $\pm \mathrm{SD}$. Non-normally distributed variables were presented as median (interquartile range).

In total, there were 3568 hospital admissions for further workup of ILD from 2000 to 2012. The number of admissions per year significantly increased from 43 in 2000 to 732 in 2012. The percentage of hospital admissions for ILD increased in the Dept of Pulmonary and Critical Care Medicine (from 2.8\% in 2000 to $10.5 \%$ of all admissions in 2012) and in the whole hospital (from $0.2 \%$ to $1.4 \%$ ).

2615 patients newly diagnosed with ILD were further analysed after exclusion of repeated admissions $(\mathrm{n}=621)$, non-Chinese ethnicity $(\mathrm{n}=3)$ and lack of critical diagnostic tools, such as high-resolution computed tomography, pulmonary function tests and blood gas analysis $(n=329)$. The number of ILD cases increased from 12 in 2000 to 532 in 2012. Similarly, the number of patients with IIP increased from nine in 2000 to 246 in 2012. The proportion of IIP to all ILD decreased from $75 \%$ to $46.2 \%$. The proportion of ILD secondary to connective tissue disease (CTD) increased from $8.3 \%$ to $28.6 \%$ of all ILD (one case in 2000 to 152 in 2012). Details of ILD subtypes and demographic characteristics of all patients are shown in table 1 . More than half $(52.3 \%)$ of ILD patients were diagnosed with IIP. In the entire cohort, the most common entity was idiopathic pulmonary fibrosis (IPF; 26.5\%), followed by CTD-ILD (24.1\%), unclassifiable IIP (13.2\%), cryptogenic organising pneumonia (8.8\%), vasculitis (6.2\%) and sarcoidosis $(5.6 \%)$.

There is limited epidemiological data about the prevalence of ILD in China [4]. Our study is the first study describing the proportion of different subtypes of ILD in China based on the current classification and definition of IIP. One major finding of our study is that hospital admissions of patients for diagnostic workup of ILD significantly increased from 2000 to 2012 in China, which has also been observed in Western countries $[5,6]$. There are several possible reasons for this observation. First, the increasing awareness on ILD and the broader access to new and more sensitive clinical tools such as high-resolution computed tomography scans has probably improved diagnostic accuracy of ILD subtypes [2]. Secondly, the older population is at higher risk of developing ILD and the Chinese population is ageing, similar to the Western population. This may have resulted in a real numerical increase of ILD. Thirdly, even if it is

@ERSpublications

Hospital admissions of patients for ILD increased in China from 2000 to 2012 and IPF was the most frequent type. This suggests an increasing recognition of ILD and/or increased burden of disease. http://ow.ly/WC6t30kWlje

Cite this article as: Ban C, Yan W, Xie B, et al. Spectrum of interstitial lung disease in China from 2000 to 2012. Eur Respir J 2018; 52: 1701554 [https://doi.org/10.1183/13993003.01554-2017]. 
TABLE 1 Interstitial lung disease (ILD): types and demographic characteristics

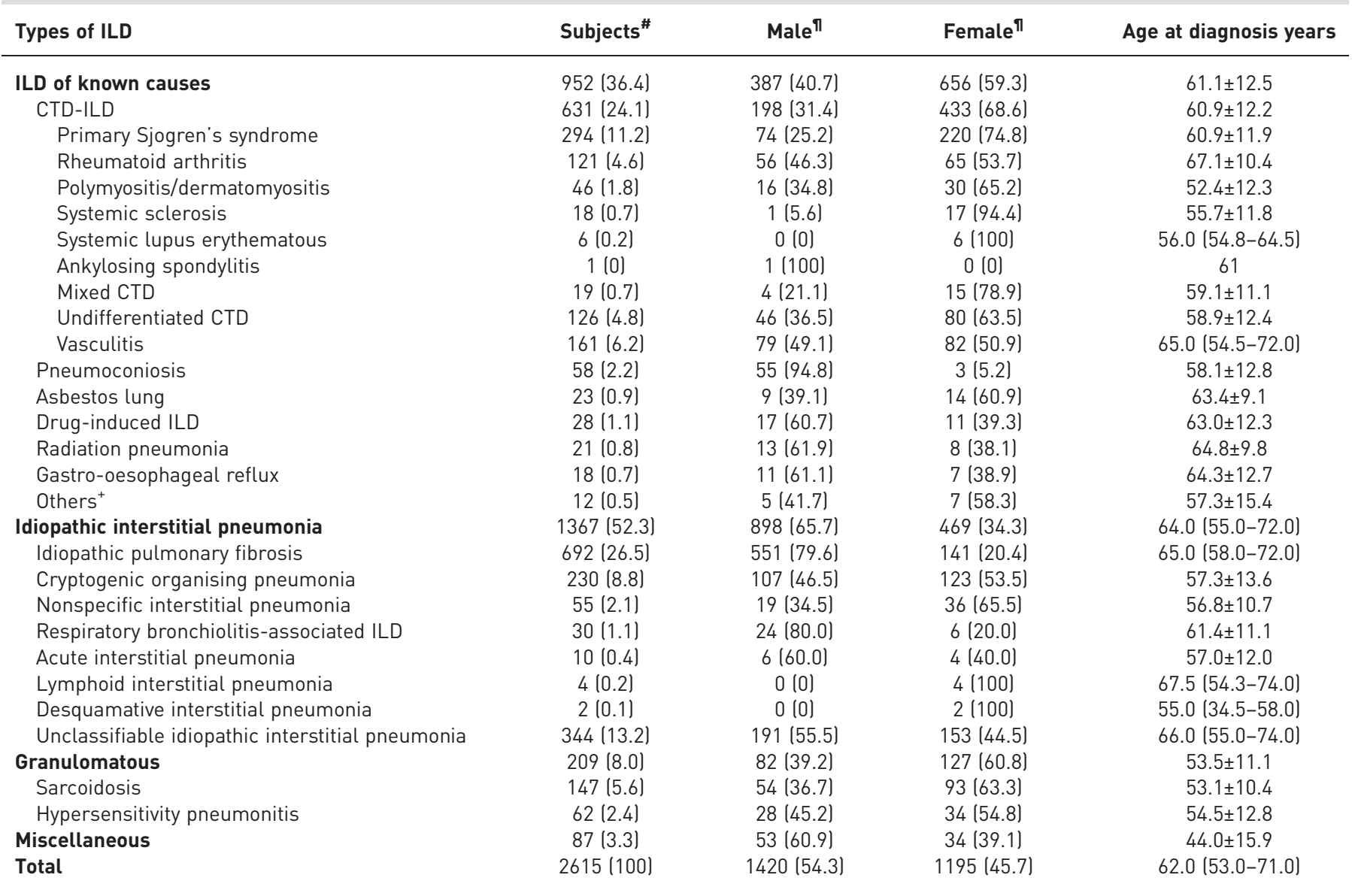

Data are presented as $\mathrm{n}(\%)$, mean \pm SD or median (interquartile range). CTD: connective tissue disease; ${ }^{\#}$ : proportion of ILD entity in all the ILD patients; ${ }^{\text {ๆ: }}$ : proportion of male or female patients with ILD subtype in the same subtype of ILD patients; ${ }^{+}$: including alveolar carcinoma and lymphangitis carcinomatosa.

speculative, the incidence of ILD in China might also have been rising because of environmental factors such as pollution, tobacco smoking, bird breeding and viral infection [3].

The distribution of ILD subtypes varies between different studies and countries because of differences in studied populations, research methods and practical approaches of establishing a clinical diagnosis. However, one consistent finding is that IPF is typically the most common subtype of ILD, which was also confirmed in our large cohort in China.

One rather unexpected finding from this study was that CTD-ILD was the second most commonly diagnosed ILD after IPF (24.1\%). The reason for this dramatic increase in patients with CTD-ILD in recent years, particularly primary Sjogren's syndrome which is the most common CTD-ILD, is unclear. We suspect that the broader awareness of ILD and a more standardised ILD diagnostic approach in China, together with the use of sensitive diagnostic technologies meant more and more ILD cases were accurately diagnosed and subtypes identified. Additionally, the majority of patients with primary Sjogren's syndrome-ILD had lung dominant diseases and came directly to our department, which might explain the higher frequency of these patients in our cohort. In our study, there were $13.2 \%$ unclassifiable ILD, which is similar to previous studies that showed that the proportion of unclassifiable ILD was between $5 \%$ and $15 \%$ [7]. The proportion of sarcoidosis was 5.6\%, which was much lower than in the USA and Europe [8-12], where sarcoidosis was the frequent entity with the proportion of $20-42 \%$. The variation of ILD subtypes in different countries is certainly affected by ethnic and geographic factors [13], besides the differences in the diagnostic awareness and approaches, and study population.

Our study has several strengths. We obtained data from a large number of patients in a major ILD centre managing Chinese patients from across the country. Furthermore, it was relatively easy to achieve a strict internal quality control through standard diagnostic protocols in a single centre. Therefore, the ILD 
spectrum in this study was more accurate and a reflection of a "real world" scenario in China. Several similar studies from previous years also reported a detailed breakdown of subtypes of ILD, but they were limited by their observational/questionnaire-based nature and would not have captured all cases like we did in our study [10-12].

Obviously, our study has several limitations. First, data were only from one major ILD centre and were retrospectively analysed, even if we collected all patients admitted with a working diagnosis of ILD. Secondly, we missed the majority of patients with pneumoconiosis because in China these patients would typically be assessed in a specialised hospital for occupational diseases. Only a prospective, multicentre study can provide a more accurate breakdown of the different ILD subtypes in China.

In summary, we showed that hospital admissions of patients for detailed workup of ILD increased in China from 2000 to 2012. IPF was the most frequent type, followed by CTD-ILD. It suggests an increasing recognition of ILD, as well as increased burden of disease.

Chengjun Ban ${ }^{1,2,5}$, Wei Yan ${ }^{1,3,5}$, Bingbing Xie ${ }^{4}$, Min Zhu ${ }^{1}$, Yan $\mathrm{Liu}^{1}$, Shu Zhang ${ }^{1}$, Qiao Ye ${ }^{1}$, Yanhong Ren ${ }^{4}$, Dingyuan Jiang ${ }^{4}$, Jing Geng ${ }^{4}$, Huaping Dai ${ }^{1,4}$ and Chen Wang ${ }^{4}$

${ }^{1}$ Dept of Pulmonary and Critical Care Medicine, Beijing Chao-Yang Hospital, Capital Medical University, Beijing, China. ${ }^{2}$ Dept of Respiration of Dongzhimen Hospital, Beijing University of Chinese Medicine, Beijing, China. ${ }^{3} \mathrm{Fu}$ Xing Hospital, Capital Medical University, Beijing, China. ${ }^{4}$ Dept of Pulmonary and Critical Care Medicine, Center of Respiratory Medicine, China-Japan Friendship Hospital, National Clinical Research Center for Respiratory Diseases, Beijing, China. ${ }^{5}$ Both contributed equally to this study.

Correspondence: Huaping Dai, Dept of Pulmonary and Critical Care Medicine, Center of Respiratory Medicine, ChinaJapan Friendship Hospital, National Clinical Research Center for Respiratory Diseases, Beijing, 100029, P.R. China. E-mail: daihuaping@ccmu.edu.cn

Received: July 212017 | Accepted after revision: July 102018

Author contributions: W. Yan contributed to the data collection and analysis and the draft of the manuscript. C. Ban contributed to the construction of the database, collection of clinical data and the revision of the manuscript. B. Xie, M. Zhu, Y. Liu, S. Zhang and Q. Ye contributed to the collection and explanation of clinical data. Y. Ren, D. Jiang and J. Geng contributed to the data analysis and the revision of manuscript. C. Weng contributed to the construction of the database, study design and the revision of the manuscript. H. Dai contributed to the construction of the ILD database, study design, the revision of the manuscript, and final approval of the version to be published. All authors read and approved the final manuscript.

Conflict of interest: None declared.

Support statement: This study was supported by the Key Program of National Natural Science Foundation of China (number 81430001) and National Key Technologies R\&D Program Precision Medicine Research (number 2016YFC0901101).

\section{References}

1 Cai M, Zhu M, Ban C, et al. Clinical features and outcomes of 210 patients with idiopathic pulmonary fibrosis. Chin Med J (Engl) 2014; 127: 1868-1873.

2 American Thoracic Society, European Respiratory Society. American Thoracic Society/European Respiratory Society International Multidisciplinary Consensus Classification of the Idiopathic Interstitial Pneumonias. Am J Respir Crit Care Med 2002; 165: 277-304.

3 Raghu G, Collard HR, Egan JJ, et al. An official ATS/ERS/JRS/ALAT statement: idiopathic pulmonary fibrosis: evidence-based guidelines for diagnosis and management. Am J Respir Crit Care Med 2011; 183: 788-824.

4 Richeldi L, Rubin AS, Avdeev S, et al. Idiopathic pulmonary fibrosis in BRIC countries: the cases of Brazil, Russia, India, and China. BMC Med 2015; 13: 237.

5 Navaratnam V, Fleming KM, West J, et al. The rising incidence of idiopathic pulmonary fibrosis in the U.K. Thorax 2011; 66: 462-467.

6 Raghu G, Chen SY, Hou Q, et al. Incidence and prevalence of idiopathic pulmonary fibrosis in US adults 18-64 years old. Eur Respir J 2016; 48: 179-186.

7 Skolnik K, Ryerson CJ. Unclassifiable interstitial lung disease: a review. Respirology 2016; 21: 51-56.

$8 \quad$ Lederer DJ, Martinez FJ. Idiopathic pulmonary fibrosis. N Engl J Med 2018; 378: 1811-1823.

9 Duchemann B, Annesi-Maesano I, Jacobe de Naurois C, et al. Prevalence and incidence of interstitial lung diseases in a multi-ethnic county of Greater Paris. Eur Respir J 2017; 50: Suppl. 57, P3366.

10 Musellim B, Okumus G, Uzaslan E, et al. Epidemiology and distribution of interstitial lung diseases in Turkey. Clin Respir J 2014; 8: 55-62.

11 Karakatsani A, Papakosta D, Rapti A, et al. Epidemiology of interstitial lung diseases in Greece. Respir Med 2009; 103: $1122-1129$.

12 Xaubet A, Ancochea J, Morell F, et al. Report on the incidence of interstitial lung diseases in Spain. Sarcoidosis Vasc Diffuse Lung Dis 2004; 21: 64-70.

13 Statement on sarcoidosis. Joint Statement of the American Thoracic Society (ATS), the European Respiratory Society (ERS) and the World Association of Sarcoidosis and Other Granulomatous Disorders (WASOG) adopted by the ATS Board of Directors and by the ERS Executive Committee, February 1999. Am J Respir Crit Care Med 1999; 160: 736-755. 